\title{
Analysis of Cortical and Mandibular Trabeculae Quality with $\beta$-crosslaps Levels in Postmenopausal Women Using Cone-beam Computed Tomography
}

\author{
Azhari Azhari ${ }^{1}$, Silviana F Diba ${ }^{2}$, Anak AlA Feranasari ${ }^{3}$, Ria N Firman ${ }^{4}$, Farina Pramanik ${ }^{5}$
}

\begin{abstract}
Aim: This study aimed to assess the correlation between cortical and mandibular trabeculae quality with beta-crosslaps ( $\beta$-CTx) levels in postmenopausal women.

Materials and methods: This study analyzed both sites of cortical and trabecular mandibular below mental foramen obtained from 17 conebeam computed tomography (CBCT) radiographs of postmenopausal women. The cortical parameters measured consisted of computed tomography index superior (CTI-S), computed tomography index inferior (CTI-I), and computed tomography mental index (CTMI); while the trabecular parameters consisted of bone volume fraction (BV/TV), trabecular thickness (Tb.Th), and trabecular space (Tb.Sp). The $\beta$-CTx level was examined, and then the results were correlated with the three parameters, each from both cortical and trabecular bones.

Results: Both trabecular $(r=0.477 ; p=0.049)$ and cortical $(r=0.411 ; p=0.038)$ parameters had positive and significant correlations $(p<0.05)$ toward the $\beta-C T x$ levels.

Conclusion: Cone-beam computed tomography was able to assess cortical bone and mandibular trabeculae quality, which was correlated with the $\beta$-CTx level of postmenopausal women.

Clinical significance: This study can be used as a consideration in detecting the decreased quality of the trabecular and cortical mandibular bones in postmenopausal women using $\mathrm{CBCT}$ and the $\beta-\mathrm{CT} x$ level before the severity occur.

Keywords: $\beta$-crosslaps, Bone quality, Cone-beam computed tomography, Postmenopausal women.

World Journal of Dentistry (2020): 10.5005/jp-journals-10015-1704
\end{abstract}

\section{INTRODUCTION}

Osteoporosis is characterized by a decrease in bone density and quality resulting in a tendency for bone fracture. Women's risk of osteoporotic fractures is higher than that of men associated with bone loss along with age. ${ }^{1}$ Women have a higher risk of osteoporosis than men, due to an increase in the estrogen hormone. The estrogen hormone in women will protect bone from losing its mass, and after menopause, there will be an increase in bone resorption, which causes postmenopausal osteoporosis. ${ }^{2}$

The dental radiograph was considered to become an alternative tool to assess bone quality by utilizing both conventional radiographic techniques and three-dimensional radiography using cone-beam computed tomography (CBCT). ${ }^{3,4}$ The advantages of using dental radiography include affordable costs and can be widely used in the population. ${ }^{3}$

Depletion of the mandibular cortical bone occurs in patients with osteoporosis; thus, a method for evaluating the mandibular cortical bone was developed in the mental foramen area. Koh and $\mathrm{Kim}^{5}$ examined cortical parameters using CBCT as a modification of the Ledgerton classification, namely computed tomography index superior (CTI-S), computed tomography index inferior (CTI-I), and the computed tomography mental index (CTMI). These parameters can be used for assessment of cortical quality in osteoporotic female patients.

Most fractures occur in the trabecular bone, so it is important to measure the trabecular bone structure as well. ${ }^{6}$ Evaluation of the bone microstructure parameter analysis through the trabeculae is essential to predict bone strength and fracture risk. ${ }^{7}$ One imaging
${ }^{1,3-5}$ Department of Dentomaxillofacial Radiology, Faculty of Dentistry, Universitas Padjadjaran, Bandung, Indonesia

${ }^{2}$ Department of Dentomaxillofacial Radiology, Faculty of Dentistry, Universitas Gadjah Mada, Yogyakarta, Indonesia

Corresponding Author: Azhari Azhari, Department of Dentomaxillofacial Radiology, Faculty of Dentistry, Universitas Padjadjaran, Bandung, Indonesia, Phone: +62 81322779900, e-mail: azhari@fkg.unpad.ac.id

How to cite this article: Azhari A, Diba SF, Feranasari AAIA, et al. Analysis of Cortical and Mandibular Trabeculae Quality with $\beta$-crosslaps Levels in Postmenopausal Women Using Cone-beam Computed Tomography. World J Dent 2020;11(2):95-98.

Source of support: Dr Azhari reported receiving research grants from Universitas Padjadjaran, Indonesia, called Internal Grant.

Conflict of interest: None

modality for analyzing the microstructural trabecular bone is $\mathrm{CBCT}$, which has a positive correlation with microcomputed tomography $(\mu \mathrm{CT})$ in trabecular analysis parameters consist of trabecular separation (Tb.Sp), trabecular thickness (Tb.Th), and total bone volume (BV/TV). Total bone volume parameters were measured by dividing voxels, which were classified as a bone with total voxels captured in one volume data. ${ }^{8}$

In addition to the radiographic examination, clinical bone quality evaluation could be examined by Beta-crosslaps ( $\beta-C T x$ ) levels. The levels of $\beta-C T x$ are the bone-specific type I collagen that describes the bone resorption process directly. ${ }^{9}$ The purpose of this 
study was to analyze the relationship of the $\beta-C T x$ levels along with mandibular bone quality of postmenopausal women using CBCT.

\section{Materials and Methods}

Cone-beam computed tomography radiographs were obtained from postmenopausal women aged 50-84 years old who attended dental treatment at Universitas Padjadjaran Dental Hospital. This study included both sides of the mandible as the region of interest (ROI); thus, there was a total of $34 \mathrm{ROI}$ samples from 17 subjects. The inclusion criteria were healthy postmenopausal women with no systemic diseases related to bone metabolism. Any criteria of oral disease such as infection, cyst, tumor, or fibro-osseous lesions were excluded either. This research was approved by the ethics committee of Universitas Padjadjaran. An informed consent was obtained from patients who agreed to be included in this study. After a CBCT examination (Picasso-Trio E-Woo Technology, Republic of South Korea), blood samples were taken the next day as they had to fast for 12 hours prior to the $\beta-C T x$ blood collection.

Three milliliters of blood was gathered from fasting subjects and stored in the vacutainer tube before centrifugated. A total of $50 \mu \mathrm{L}$ of serum was taken after blood was centrifuged for 15 minutes at 3,000 rpm. Blood serum was stored in cup samples at $2-8^{\circ} \mathrm{C}$ before analysis. ${ }^{10}$ Samples were analyzed no more than 3 days after sampling. The $\beta-C T x$ level was analyzed using electrochemiluminescence immunoassay (ECLIA) with the Elecsys 2010 (Roche Diagnostic, USA) based on the manufacturer's preferences and laboratory quality-control procedures.

\section{Trabecular Quality Assessment}

In the axial view, the axis was directed so that the mental foramen could be seen in the coronal view, as shown in Fig. 1D. A squareshaped ROI sized $3 \times 3 \mathrm{~mm}$ was placed in the trabecular area inferior to the mental foramen. That ROI was analyzed using the BoneJ software (version 1.4.2, National Institutes of Health, Bethesda, USA) with features of bone fraction and thickness. The percentage of bone mass (BV/TV), trabecular thickness (Tb.Th), and the distance between trabeculae (Tb.Sp) were analyzed as trabecular quality.

\section{Cortical Quality Assessment}

Mandibular cortical parameters were analyzed in the same field of view as the trabecular, which was from a coronal view (Figs 1A-C).
Cortical parameters analyzed were "CTI-S," "CTI-I," and "CTMI," using the method from Koh and Kim. ${ }^{5}$ Computed tomography mandibular index superior (CTI-S) was the ratio of cortical width $(W)$ to the distance of the superior margin of the mental foramen to the inferior border of the mandible (S). Computed tomography mandibular index inferior (CTI-I) was a ratio of cortical width (W) to the distance of the inferior margin of the mental foramen to the inferior border of the mandible $(I)$. The computed tomography mental index (CTMI) was the width of the inferior mandibular border $(W)$; each calculated with equations below:

$\mathrm{CTI}-\mathrm{S}=W / \mathrm{S}$

$\mathrm{CTI}-\mathrm{I}=W / \mathrm{I}$

$\mathrm{CTMI}=W$

\section{Results}

A descriptive data of the $\beta$-Ctx level, trabecular parameters, and cortical parameters are shown in Tables 1 and 2 . Subjects were divided into three age groups to facilitate the presentation of descriptive data. The group range was 55-64 y/o, 65-74 y/o, and $75-84$ y/o while the sample size was 10,3 , and 4 people, respectively.

The highest levels of $\beta-C t x$, Tb.Th, and Tb.Sp were found in the $65-74$ years old age group, with respective values of $0.525,1.137$, and 0.996 . The highest percentage of BV/TV was 0.426 , which was found in the age group 75-84 years old, as seen in Table 1. In a study conducted by Monje et al., ${ }^{11}$ the mean normal values of posterior mandibular trabecular parameters among adult women in Shanghai were $0.400,0.300$, and 0.370 for BV/TV, Tb.Th, and Tb.Sp values, respectively.

Based on Table 2, the age group of 75-84 years old has the highest average of CTI-I, CTI-S, and CTMI, with the values of 0.235 , 0.271 , and 3.467, respectively. The average normal values of CTI-I, CTI-S, and CTMI were $0.250,0.210$, and 3.220 , respectively according to a study on postmenopausal women in South Korea. ${ }^{5}$

The trabecular parameters consisted of BV/TV, Tb.Th, and Tb.Sp was correlated positively $(r=0.477)$ to the level of $\beta-C t x$, as well as the cortical parameters $(r=0.411)$, consisted of CTI-S, CTI-I, and CTMI. Trabecular and cortical parameters measured using CBCT showed a positive and significant correlation $(p<0.05)$ related to $\beta$-Ct $x$ levels in postmenopausal women (Table 3 ).
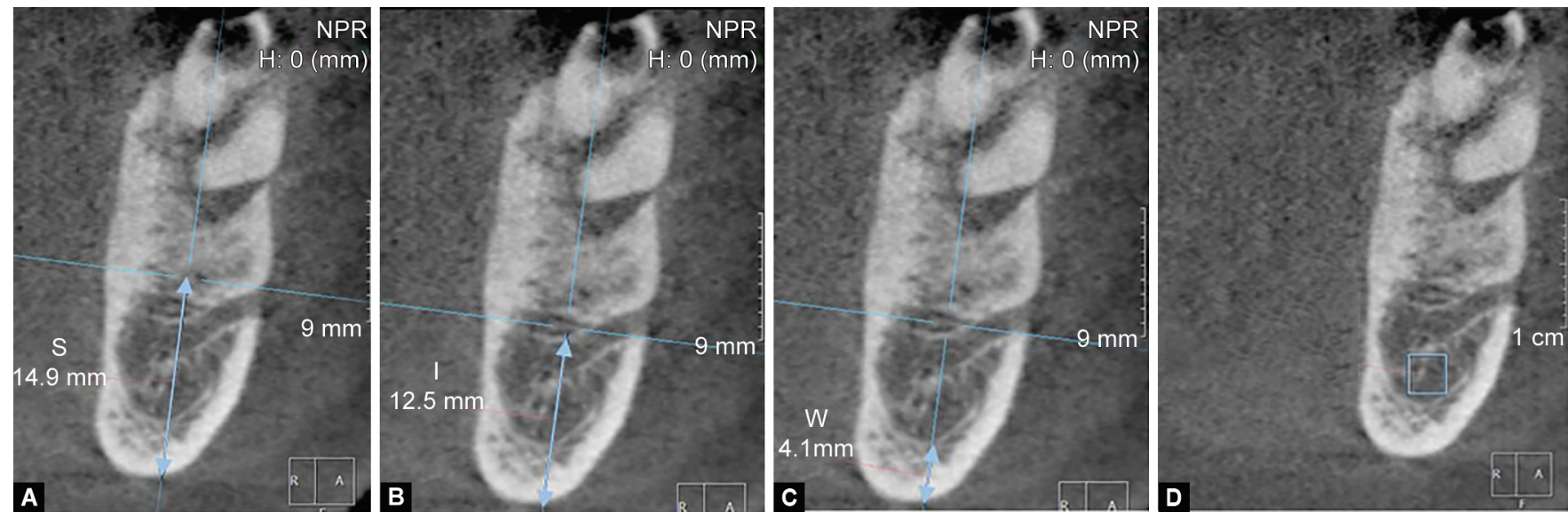

Figs $1 \mathrm{~A}$ to $\mathrm{D}$ : The mental foramen region observed from the coronal view: (A) Distance from the superior mental foramen to the inferior border of the mandibular cortical; (B) Distance from the inferior mental foramen to the inferior border of the mandibular cortical; (C) Mandibular cortical thickness measured; (D) The region of interest sized $3 \times 3 \mathrm{~mm}$ between the mental foramen and the mandibular cortical 
Analysis of Cortical and Mandibular Trabeculae Quality with $\beta$-crosslaps Levels in Postmenopausal Women

Table 1: Descriptive data of the $\beta$-Ctx level with trabecular parameters BV/TV, Tb.Th., and Tb.Sp based on the age group

\begin{tabular}{|c|c|c|c|c|c|c|c|c|c|}
\hline \multirow[b]{2}{*}{ Age } & \multirow[b]{2}{*}{$n$} & \multicolumn{2}{|c|}{$\beta-C T x(n g / m L)$} & \multicolumn{2}{|c|}{ BV/TV (\%) } & \multicolumn{2}{|c|}{ Tb.Th (mm) } & \multicolumn{2}{|c|}{$T b . S p(m m)$} \\
\hline & & Mean & $S D$ & Mean & $S D$ & Mean & $S D$ & Mean & $S D$ \\
\hline $55-64$ & 10 & 0.461 & 0.198 & 0.406 & 0.082 & 1.122 & 0.334 & 0.847 & 0.280 \\
\hline $65-74$ & 3 & 0.525 & 0.010 & 0.408 & 0.122 & 1.137 & 0.210 & 0.996 & 0.331 \\
\hline $75-84$ & 4 & 0.480 & 0.197 & 0.426 & 0.048 & 1.105 & 0.125 & 0.946 & 0.150 \\
\hline
\end{tabular}

BV/TV, bone volume/tissue volume; Tb.Th, trabecular thickness; Tb.Sp, trabecular separation; SD, standard deviation

Table 2: Descriptive data of the $\beta$-Ctx level with cortical parameters CTI (S), CTI (I), and CTMI based on the age group

\begin{tabular}{|c|c|c|c|c|c|c|c|c|c|}
\hline \multirow[b]{2}{*}{ Age } & \multirow[b]{2}{*}{$n$} & \multicolumn{2}{|c|}{$\beta-C T x(n g / m L)$} & \multicolumn{2}{|c|}{$\mathrm{CTI}(\mathrm{S})(\mathrm{mm})$} & \multicolumn{2}{|c|}{$\mathrm{CTI}(\mathrm{I})(\mathrm{mm})$} & \multicolumn{2}{|c|}{ CTMI $(\mathrm{mm})$} \\
\hline & & Mean & $S D$ & Mean & $S D$ & Mean & $S D$ & Mean & $S D$ \\
\hline $55-64$ & 10 & 0.461 & 0.198 & 0.212 & 0.041 & 0.244 & 0.046 & 3.186 & 0.599 \\
\hline $65-74$ & 3 & 0.525 & 0.010 & 0.204 & 0.138 & 0.240 & 0.150 & 3.050 & 2.264 \\
\hline $75-84$ & 4 & 0.480 & 0.197 & 0.235 & 0.013 & 0.271 & 0.013 & 3.467 & 0.344 \\
\hline
\end{tabular}

CTI-S, computed tomography index superior; CTI-I, computed tomography index inferior; CTMI, computed tomography mental index; SD, standard deviation

Table 3: Results of the correlation analysis between levels of $\beta$-Ctx with each cortical and trabecular parameter

\begin{tabular}{llll}
\hline Parameter & $R$ & R square & $p$ value \\
\hline $\begin{array}{l}\beta-C T x \text { and trabecular parameter } \\
\text { (BV/TV, Tb.Th., Tb.Sp.) }\end{array}$ & 0.477 & 0.227 & $0.049^{\mathrm{a}}$ \\
$\begin{array}{l}\beta-C T x \text { and cortical parameter (CTI-S, } \\
\text { CTI-I and CTMI) }\end{array}$ & 0.411 & 0.169 & $0.038^{\mathrm{a}}$ \\
\hline
\end{tabular}

${ }^{\mathrm{a}} p<0.05$

\section{Discussion}

This study used CBCT as a noninvasive technique for assessing bone quality through the three-dimensional shape of the bone, bone density, and the microstructural analysis correlated with $\beta-C T x$ levels in postmenopausal women. This clinical parameter was used because it is the first bone turnover marker (BTM) in the serum, which is easily examined using automatic tools and does not require additional creatinine examination. ${ }^{10}$ Beta-CTx was not degraded in the blood or excreted by the kidneys so that CTx directly reflects bone matrix collagen degradation, which associated to bone resorption histomorphometric parameters. ${ }^{12,13}$ Postmenopausal women tend to have a reduction of low bone mass related to osteoporosis. This silent disease would not present its symptoms until severity occurs, leading to fracture, disability, and even mortality. ${ }^{14}$ Therefore, a dental radiograph is considered to become a common diagnostic tool to predict bone quality reduction. By detecting these bone structure alterations, the dentist could refer postmenopausal patients for further examination before the severity occurs. ${ }^{15}$ Trabecular parameter assessment using CBCT could also estimate bone density prior to implant placement. Bone density was related to the implant success since most of dental implant failures occurred in bone with low density. ${ }^{16}$

Various methods can be applied to assess bone quality using dental radiography. Bone density could be assessed from a periapical radiograph and aluminum step wedge. Amam and Rustom ${ }^{17}$ found that mandibular alveolar bone density had a significant correlation with bone mineral density (BMD) of the lumbar spine and the femoral neck in osteoporosis. ${ }^{17}$ The dental radiograph image analysis of fractal dimension could depict bone quality and its increase showing reduced BMD in osteoporosis patients. $^{14}$

In this study, the $\beta-C T x$ levels of postmenopausal women in 65-74 years old and 75-84 years old groups were 0.585 and 0.480 $\mathrm{ng} / \mathrm{mL}$, respectively. Beta-CTx as the marker of bone resorption decrease with age, then increase sharply in women over 50 years old and begin to decline again in women over the age of $70 .{ }^{12}$ In a study conducted by Halimah et al. in Indonesia, the means of the $\beta-C T x$ level in normal, osteopenia, and osteoporotic postmenopausal women were $0.303,0.454$, and $0.558 \mathrm{ng} / \mathrm{mL}$, respectively. ${ }^{10}$ Meanwhile, in Australia, the interval of the $\beta-C T x$ level for women aged 50 and above was $0.1-0.7 \mathrm{ng} / \mathrm{mL} .{ }^{13}$ Histomorphometric studies showed a decrease in bone resorption, bone formation, and cancellous bone volume (BV/TV) along with age. The BV/TV value in women experience a downward trend after 50 years, which is associated with the onset of menopause. ${ }^{13}$ Many factors influence the value of bone turnover markers to vary from country, including age, weight, physical activity, and gender. ${ }^{12,13}$

The measurement result of the mandibular cortical bone height with CBCT-3D radiographs correlates well with the measurement of the panoramic mandibular index (PMI) and the mental index (MI) on a panoramic radiograph. ${ }^{18}$ A study conducted by Koh and $\mathrm{Kim}^{5}$ showed that there was a significant correlation of CTI (S) measurement with $\mathrm{CTI}$ (I) on CBCT-3D radiographs in an assessment of osteoporotic women. Research conducted by Mostafa et al. ${ }^{19}$ also showed a significant correlation in the $\mathrm{MI}$ and $\mathrm{PMI}$ on panoramic radiographs. In this study, cortical parameters had a significant positive correlation toward the $\beta-C T x$ level. Mandible cortical assessment is not only done quantitatively but also qualitatively through a panoramic radiograph using Klemetti index (KI). The cortical sharpness is divided into three visual scales, which range from clear to porous cortical border. ${ }^{15}$

A study conducted by Azhari et al. ${ }^{20}$ showed that there was a significant correlation between the mandibular cortical bone height and $\beta-C T x$ levels using a panoramic radiograph to assess osteoporosis. Abnormalities of the bone remodeling process can result in changes of the bone formation and resorption, where bone resorption was characterized by the $\beta$-CTx levels. ${ }^{20}$

In the trabecular section, three parameters consisted of BV/TV, $\mathrm{Tb} . \mathrm{Th}$, and Tb.Sp had a significant correlation with the $\beta$-CTx level. 
These results indicated that analyzing bone quality using $\mathrm{CBCT}$ in association with the value of $\beta-C T x$ requires a combination of these three microstructural parameters. According to Vasikaran et al., ${ }^{21}$ the BV/TV and Tb.Th showed the bone density value, while the Tb.Sp was contrasted with bone density parameters; thus, changes in the bone mass were affected by the balance between bone resorption and formation which decreased with age. The increasing value of Tb.Sp indirectly indicated an imbalance between bone resorption and formation, which was the resorption speed was to be higher than the bone formation speed and contributed to the loss of bone mass. ${ }^{10,22}$ Besides osteoporosis, CBCT could assist the assessment of the trabecular parameter in patients with bonerelated diseases, such as osteonecrosis and temporomandibular joint osteoarthritis. ${ }^{23,24}$

The limitation of this study includes a small sample size. Besides, software tools only could display the slices of image CBCT where the cortical and trabecular parameters were measured. $\mathrm{A}$ better software to extract trabecular structures from $C B C T$ and correlation between the parameters with other bone turnover markers might be done in the future.

\section{Conclusion}

Mandibular cortical and trabecular parameters on $\mathrm{CBCT}$ radiographs correlated with the $\beta-C T x$ level in postmenopausal women. CBCT-3D radiographs had high accuracy and resolution, thus can be used to detect the mandibular cortical bone height. The $\beta-C T x$ level is a biomarker of bone resorption, which depicted bone quality decrease in postmenopausal women. Cone-beam computed tomography can be used to assess the quality of the cortical bone and the mandibular trabeculae associated with the $\beta$-CTx level in postmenopausal women as early detection of decreasing bone quality.

\section{Ethical Approval}

This research has been approved by the ethics commission of the Universitas Padjadjaran Indonesia (No. 941/UN6.KEP/EC/2018).

\section{Author Contributions}

Study concept and design; critical revision of the manuscript for important intellectual content; administrative, technical, and material support; and study supervision were contributed by Azhari, Firman, and Pramanik. Acquisition of data was contributed by Diba, Feranasari, and Pramanik. Analysis and interpretation of data, drafting of the manuscript, and statistical analysis were contributed by Diba and Feranasari.

\section{References}

1. Paramashivaiah R, Padmanabhan S, Dwarakanath C, et al. Periodontal status of postmenopausal women with osteoporosis. World J Dent 2011;2(4):297-301. DOI: 10.5005/jp-journals-10015-1101.

2. Bajoria $A$, Babshet $M$, Patil P. Bone mineral density measurement of the jaws - a review. J Investig Dent Sci 2014;1(1):1-8.

3. Verheij JGC, Geraets WGM, Van Der Stelt PF, et al. Prediction of osteoporosis with dental radiographs and age. Dentomaxillofacial Radiol 2009;38(7):431-437. DOI: 10.1259/dmfr/55502190.

4. Barngkgei I, Haffar IAL, Khattab R. Osteoporosis prediction from the mandible using cone-beam computed tomography. Imaging Sci Dent 2014;44(4):263-271. DOI: 10.5624/isd.2014.44.4.263.
5. Koh KJ, Kim KA. Utility of the computed tomography indices on cone beam computed tomography images in the diagnosis of osteoporosis in women. Imaging Sci Dent 2011;41(3):101-106. DOI: 10.5624/isd.2011.41.3.101.

6. Meiyanti M. Epidemiology of osteoporosis in postmenopausal women aged 47 to 60 years. Universa Med 2010;29(3):169-176.

7. Brandi ML. Microarchitecture, the key to bone quality. Rheumatol (United Kingdom) 2009;48(Suppl 4):iv3-8. DOI: 10.1093/ rheumatology/kep273.

8. Klintström E. Image analysis for trabecular bone properties on conebeam CT data. 2017 (No. 1594).

9. Chubb SAP. Measurement of C-terminal telopeptide of type I collagen (CTX) in serum. Clin Biochem 2012;45(12):928-935. DOI: 10.1016/ j.clinbiochem.2012.03.035.

10. Halimah E, Rositawati W, Pratiwi I. N-MID Osteocalcin (N-MID oc) and $\beta$-crosslaps ( $\beta$-CTX) as Biochemical markers of bone turnover in menopausal women. Indones J Clin Pharm 2016;5(1):67-74. DOI: 10.15416/ijcp.2016.5.1.67.

11. Monje $\mathrm{A}, \mathrm{Wu} \mathrm{Y}$, Huang $\mathrm{W}$, et al. Influence of posterior mandibular dimensions on alveolar bone microarchitecture. Int J Oral Maxillofac Implants 2017;32(2):423-430. DOI: 10.11607/jomi.5144.

12. Hu WW, Zhang Z, He JW, et al. Establishing reference intervals for bone turnover markers in the healthy shanghai population and the relationship with bone mineral density in postmenopausal women. Int J Endocrinol 2013;2013:1-7. DOI: 10.1155/2013/513925.

13. Jenkins $N$, Black M, Paul E, et al. Age-related reference intervals for bone turnover markers from an Australian reference population. Bone 2013;55(2):271-276. DOI: 10.1016/j.bone.2013.04.003.

14. Kavitha M, Khan M, Vijayalakshmi KR. Fractal dimension analysis in digital periapical radiographs: a diagnostic indicator of osteoporosis in post-menopausal women. J Indian Acad Oral Med Radiol 2017;29(2):84-89. DOI: 10.4103/jiaomr.JIAOMR_137_16.

15. Graham J. Detecting low bone mineral density from dental radiographs: a mini-review. Clin Cases Miner Bone Metab 2015;12(2):178-182. DOI: 10.11138/ccmbm/2015.12.2.178.

16. González-García R, Monje F. The reliability of cone-beam computed tomography to assess bone density at dental implant recipient sites: a histomorphometric analysis by micro-CT. Clin Oral Implants Res 2012. 1-9.

17. Amam A, Rustom J. Assessment of mandibular alveolar bone density in osteoporotic adults in Syria. Open J Dent Oral Med 2014;2(2):26-32.

18. Benson BW, Prihoda TJ, Glass BJ. Variations in adult cortical bone mass as measured by a panoramic mandibular index. Oral Surg Oral Med Oral Pathol Oral Radiol 1991;71(3):349-356. DOI: 10.1016/00304220(91)90314-3.

19. Mostafa RA, Arnout EA, Fotouh MMA. Feasibility of cone beam computed tomography radiomorphometric analysis and fractal dimension in assessment of postmenopausal osteoporosis in correlation with dual X-ray absorptiometry. Br Inst Radiol 2016(7). DOI: 10.1259/dmfr.20160212.

20. Azhari, Epsilawati L, Sam B, et al. Assessment of Mandible Cortical Highness correlation with CTx and Osteocalcin level and description of trabecular pattern in neck of condily In Panoramic Radiography to detect osteoporosis. 2013.

21. Vasikaran $S$, Eastell $R$, Bruyère $O$, et al. Markers of bone turnover for the prediction of fracture risk and monitoring of osteoporosis treatment: a need for international reference standards. Osteoporos Int 2011;22(2):391-420. DOI: 10.1007/s00198-010-1501-1.

22. Dos Reis LM, Batalha JR, Muñoz DR, et al. Brazilian normal static bone histomorphometry: effects of age, sex, and race. J Bone Miner Metab 2007;25(6):400-406. DOI: 10.1007/s00774-007-0778-4.

23. Liang X, Liu S, Qu X, et al. Evaluation of trabecular structure changes in osteoarthritis of the temporomandibular joint with cone beam computed tomography imaging. Oral Sur Oral Med Oral Pathol Oral Radiol 2017;124(3):315-322. DOI: 10.1016/j.0ooo.2017.05.514.

24. Pham D, Jonasson G, Kiliaridis S. Assessment of trabecular pattern on periapical and panoramic radiographs: a pilot study. Acta Odontol Scand 2010;68(2):91-97. DOI: 10.3109/00016350903468235. 